\title{
Evaluating the Risk of Muscle Injury in Football-kicking Training with OpenSim
}

\author{
Jing Chang ${ }^{1}$, Wenrui Wang ${ }^{2}$, Damien Chablat ${ }^{3}$, and Fouad Bennis ${ }^{4}$ \\ ${ }^{1}$ Tsinghua University, Beijing, 100084, China \\ les_astres@tsinghua.edu.cn \\ ${ }^{2}$ École Centrale de Nantes, Nantes 44300, France \\ wwr.1122@163.com \\ ${ }^{3}$ Laboratoire des Sciences du Numérique de Nantes, UMR CNRS 6004, Nantes \\ 44300, France \\ damien.chablat@cnrs.fr \\ ${ }^{4}$ École Centrale de Nantes, LS2N, UMR CNRS 6004, Nantes 44300, France \\ fouad.bennis@ec-nantes.fr
}

\begin{abstract}
Football, as the most popular sport in the world, has a very wide spread worldwide. With the wide spread of football, more and more people participate in football activities. Football is a very strenuous sport and athletes are easily injured. This article examines the problem of kicking through two experiments, one is the normal kicking process, and the other is the process of imitating the kicking. The two sets of experiments use the same motions, but their applied external forces are different. In the normal kicking, the football will receive a force on the foot during the kicking process. Conversely, in the imitation kicking, the foot is not in contact with the football and no extra force is apply on the foot. Analyze the impact of football on the body by comparing the above two experiments. OpenSim software is used to calculate the forces of each muscle by simulating the process of kicking a ball to analyze the muscle fatigue. It is judged that those muscles are vulnerable to injury during the kick. Athletes should focus on strengthening the exercise of these muscles, increase the strength of related muscles, and focus on protecting related muscles during exercise to reduce injuries.
\end{abstract}

Keywords: Football, football injury, football kicking, OpenSim, muscle fatigue, fatigue model

\section{Introduction}

As one of the most popular and physically demanding games around the world, football is reported to bring about 12 to 35 injury incidences per 1000 hours outdoor games [1]. Most of the injury is caused by impact trauma [1], followed by overuse injury, which takes $34 \%$ of all [2]. Researches on retired football players have shown that they are faced with twice the incidence of coxarthrosis compared with normal 
people [3, 4]. In fact, the lifelong occurrence of musculoskeletal problems in former football athletes is reported to be even higher than that of former long-distance runners or shooters [5].

Kicking football is a basic training specific for football players. A normal football, weighing about $0.44 \mathrm{~kg}$ could reach up to $200 \mathrm{~km} / \mathrm{h}$ in a shot, which may bring heavy load to athletes. Body parts that form the kicking-related kinetic chain such as the back, hip, and knee are reported to be the most fragile parts in terms of musculoskeletal injuries [2]. To prevent related impact and overuse injuries, it is necessary to have an evaluation on the muscle loads as well as a prediction on the fatigue process in football-kicking training.

OpenSim is one of the main biomechanical human modeling platforms [6]. Various kinematical and dynamical calculations are available to simulate human motion as well as to estimate body loads. Furthermore, algorithms such as Static optimization (SO), Compute muscle control (CMC) were developed to estimate the activities of individual muscles [6]. In previous researches, the OpenSim platform has been used to study muscle contributions to sports such as running [7], to determine the risky muscle groups in physical works such as overhead drilling [8], and to study the ways of human-machine interaction from the aspects of body load distribution, such as to compare the unilateral and bilateral crutch gaits [9]. OpenSim will be effective to study the muscle contribution in football-kicking.

Muscle fatigue is an important contributing factor to overuse muscle injuries as well as musculoskeletal disorders. To date, very few studies have investigated the muscle fatigue induced by football-kicking. The muscle fatigue process has been described by the decline of muscle maximal voluntary contraction force (MVC) [10]. As illustrated by the Dynamic muscle fatigue model, the rate of force decline is dependent on the current muscle loads as well as the subject-specific trait. By the time, the muscle MVC declines to a level close to its current load, the risk of overuse injury increases.

In this study, we seek to understand how different muscle groups contribute to football-kicking training as well as to predict the fatigue process in this training. Null hypotheses include:

1) Muscle groups near the acting point of external force (i.e., the calf and knee) contribute more than that of the farther (i.e., the hip and trunk muscles);

2) Muscles of left and right sides differ in contribution. The dominant side that applies football-kicking contributes more than the other.

3) The lower leg and knee muscles contribute especially to accelerating the football.

Results of this study will expand our knowledge about the kinetics of footballkicking and help prevent both impact and overuse injuries in football.

\section{Methods}

This is a biomechanical simulation study. A football-kicking motion is simulated and muscle activations with and without the action force of football are calculated with OpenSim. Muscle fatigue process is indicated by muscle MVC declines in constant repetitive football-kicking training. 


\subsection{OpenSim model}

In this study, a generic OpenSim model, gait2354 is used for simulation. It is one of the most widely-used models with satisfactory effectiveness (for details see https://simtk-

confluence.stanford.edu/display/OpenSim/Gait+2392+and+2354+Models). Muscles are categorized by four groups. The trunk group includes three pairs of muscle (the internal and external obliques, and the erector spine); the hip group includes 18 pairs of muscle that connects the pelvis and leg; the knee group include seven pairs of muscle that cross the knee joint; the calf group includes 4 pairs of muscles.

\subsection{Data and simulation}

For kicking football, athlete lifts the dominant foot and swings forward to reach the ball, during which ground reaction force acts on the supporting foot and footballaccelerating force on the dominant foot. In this study, we compare the case with or without the accelerating force. Motion and force data come from OpenSim document (https://simtk.org/frs/index.php?group_id=679, copyright open from Stanford University), which represents a subject kicking football with the right foot. The kicking motion lasts for 1.5 second.

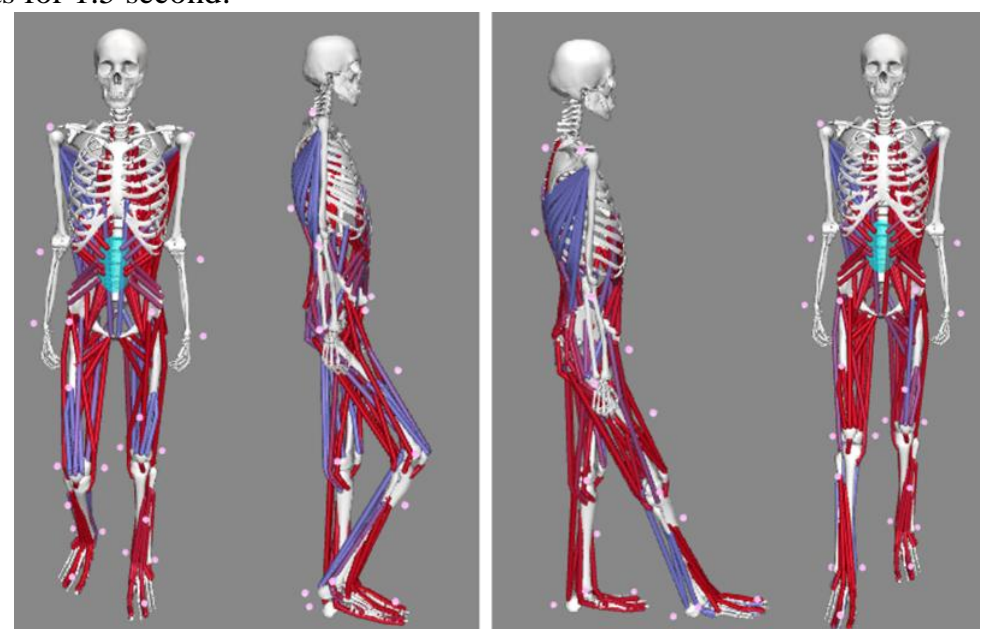

Figure 1. The simulated football-kicking motion at $\mathrm{t}=0.45 \mathrm{~s}$ (left) and $\mathrm{t}=0.9 \mathrm{~s}$ (right).

Muscle contribution is indicated by computed muscle activation. Inverse kinematics and inverse dynamics are computed to estimate joint positions and joint moments; then Residual reduce algorithm and CMC are applied to estimate the muscle activations. 


\subsection{Muscle fatigue analysis}

The Dynamic muscle fatigue model is presented as Eq.1. In this study, we approximate the maximal muscle ability $\left(F_{\max }(t)\right)$ as the MVC. With the computed muscle activation from OpenSim simulation, the profile of maximal muscle force is predictable. As illustrated in Introduction, the muscle would be at risk for overuse injury by the time its maximal force declines near the required muscle load. In this study, we analyze the muscle fatigue process in repetitive football-kicking motions to check athlete's maximal endurance time. The subject-specific muscle fatigability is set to 1 $\mathrm{min}^{-1}$, indicating average fatigue resistance [8].

$$
\frac{d F(t)}{d t}=-k \frac{F_{\text {load }}(t)}{F_{\max }(t)} F(t)
$$

With

- $F_{\text {cem }}(\mathrm{t}) \quad \mathrm{N} \quad$ Current existing maximal muscle ability

- $F_{\text {load }}(\mathrm{t}) \mathrm{N} \quad$ Muscle load

- $F_{\max }(\mathrm{t}) \quad \mathrm{N} \quad$ Maximal muscle ability

- $k \quad \min ^{-1} \quad$ Subject-specific fatigability

- $F(\mathrm{t}) \quad 1 \quad$ Muscle activation

\section{Results}

\subsection{Muscle contribution in football-kicking}

The estimated muscle activations are summarized in Table 1. In general, muscle group that contribute the most are the knee and the hip, instead of the calf; the first hypothesis is not supported. Muscles on the right side (dominant side in this simulation) are more activated, which supports the second hypothesis. However, significant difference only presents in the knee and calf. Finally, contrary to the third hypothesis, acting force from football only makes significant difference in the trunk muscle group. Both the left and right trunk are much more activated in simulation with football (2.18\% vs. $24 \%$ ), suggesting that football acceleration force mainly depends on the trunk muscles.

Table 1. The calculated muscle activations in football-kicking training. Muscles are compared between left and right side.

\begin{tabular}{|c|c|c|c|c|c|c|}
\hline \multirow{2}{*}{$\begin{array}{l}\text { Muscle } \\
\text { group }\end{array}$} & \multirow{2}{*}{$\begin{array}{l}\text { Number } \\
\text { muscles }\end{array}$} & & \multicolumn{4}{|c|}{ Muscle activation (\%) } \\
\hline & & & $\begin{array}{l}\text { R-with } \\
\text { football }\end{array}$ & $\begin{array}{l}\text { R-without } \\
\text { football }\end{array}$ & $\begin{array}{l}\text { L-with } \\
\text { football }\end{array}$ & $\begin{array}{l}\text { L-without } \\
\text { football }\end{array}$ \\
\hline Hip & 18 & & 35.64 & 36.77 & 33.23 & 32.11 \\
\hline Knee & 7 & & 40.54 & 41.53 & 28.65 & 27.20 \\
\hline Calf & 4 & & 18.22 & 17.49 & 6.85 & 5.48 \\
\hline Trunk & 3 & & 2.18 & 24.21 & 2.18 & 23.97 \\
\hline
\end{tabular}




\subsection{Fatigue process of football-kicking training}

\subsubsection{The muscle force profiles}

Muscles that activated the most in each muscle group are chosen to study the fatigue process. They are the iliacus on the hip, the biceps femoris-long head (bifemih) on the knee, the tibialis anterior (tib_ant) of the calf, and the internal oblique (intobl) of the trunk. Mean activation of these muscles are shown in Table 2, and their force profiles are presented in Figure 2.

Table 2. The typical muscle activation of each muscle group in football-kicking training.

\begin{tabular}{cccccc}
\hline $\begin{array}{c}\text { Muscle } \\
\text { group }\end{array}$ & $\begin{array}{l}\text { Name of } \\
\text { muscle }\end{array}$ & $\begin{array}{c}\text { R-with } \\
\text { football }\end{array}$ & $\begin{array}{c}\text { R-without } \\
\text { football }\end{array}$ & $\begin{array}{c}\text { L-with } \\
\text { football }\end{array}$ & $\begin{array}{c}\text { L-without } \\
\text { football }\end{array}$ \\
\hline Hip & Iliacus & 67.1 & 74.9 & 65.0 & 65.5 \\
Knee & Bifemih & 35.8 & 33.1 & 25.9 & 26.9 \\
Calf & Tib_ant & 31.8 & 32.3 & 15.0 & 8.9 \\
Trunk & Intobl & 2.1 & 29.8 & 2.1 & 29.1 \\
\hline
\end{tabular}
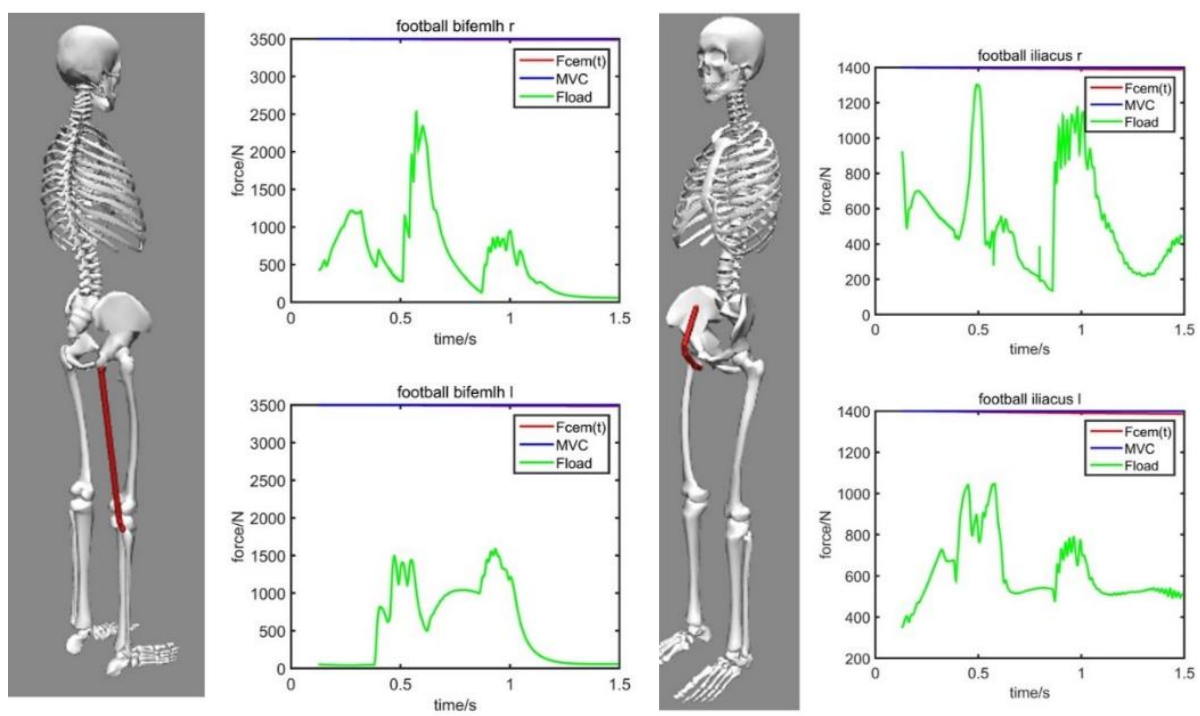

(a)

(b) 

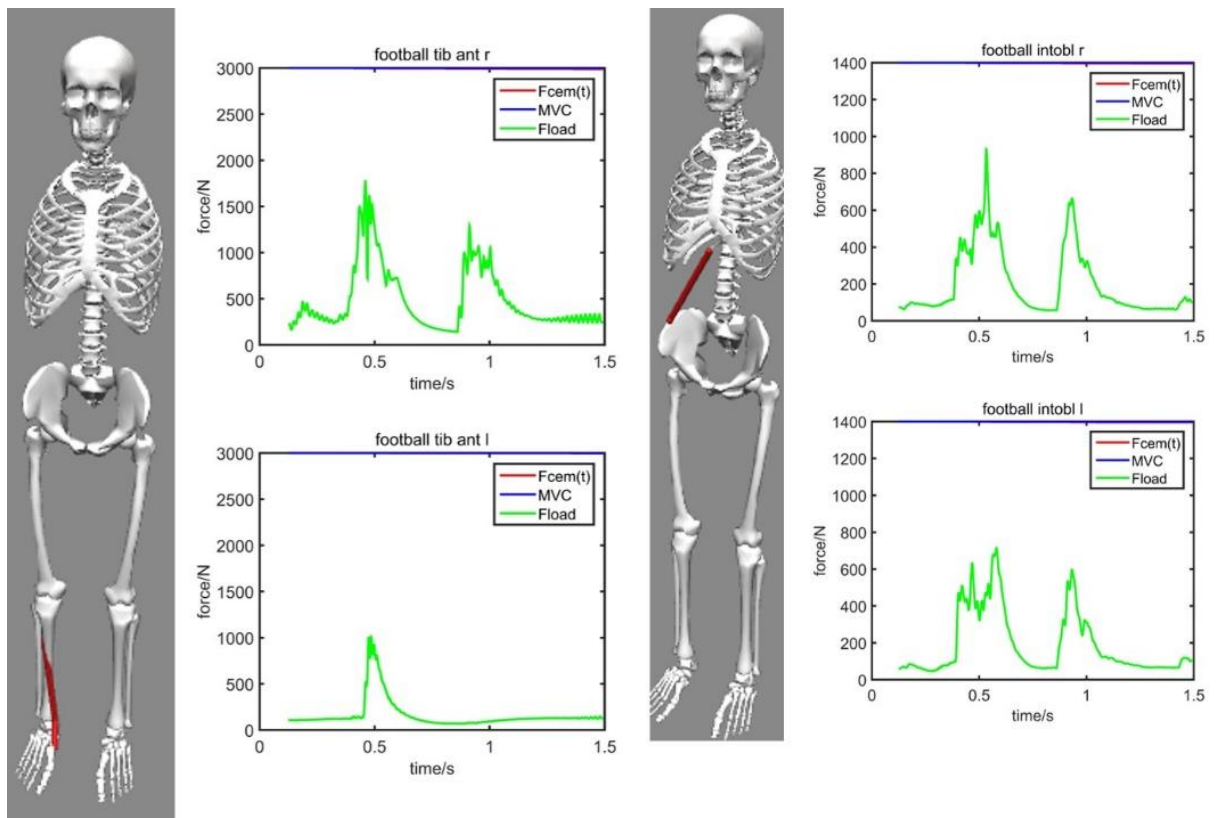

(c)

(d)

Figure 2. The estimated muscle exertions in a football-kicking motion. (a) iliacus of the hip; (b) biceps femoris-long head (bifemlh) of the knee; (c) tibialis anterior (tib_ant) of the calf; (d) internal oblique (interobl) of the trunk.

\subsubsection{The fatigue process and maximal endurance time}
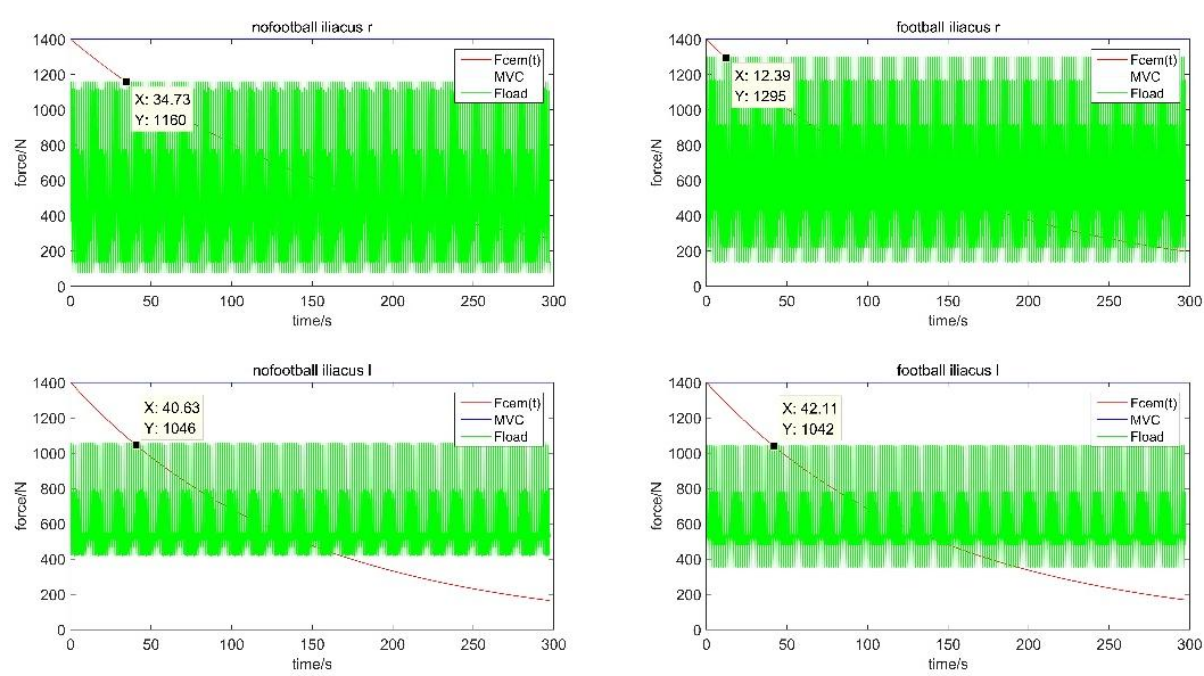

Figure 3. The fatigue process of right and left iliacus on the hip. 

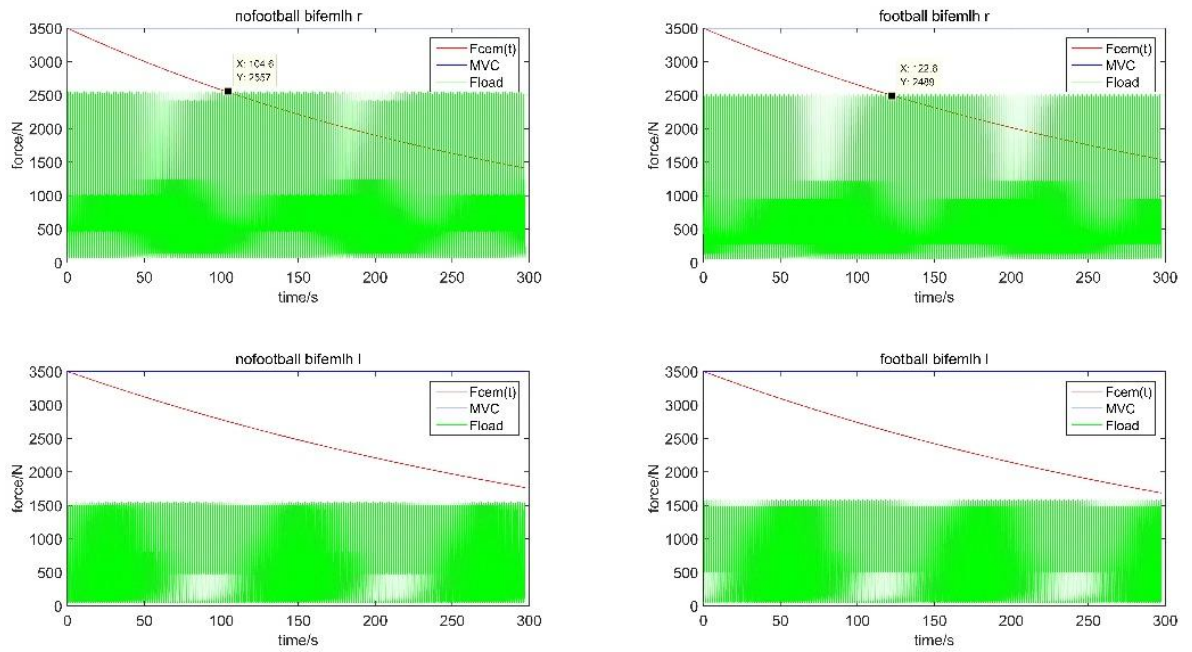

Figure 4. The fatigue process of right and left bifemih of the knee.
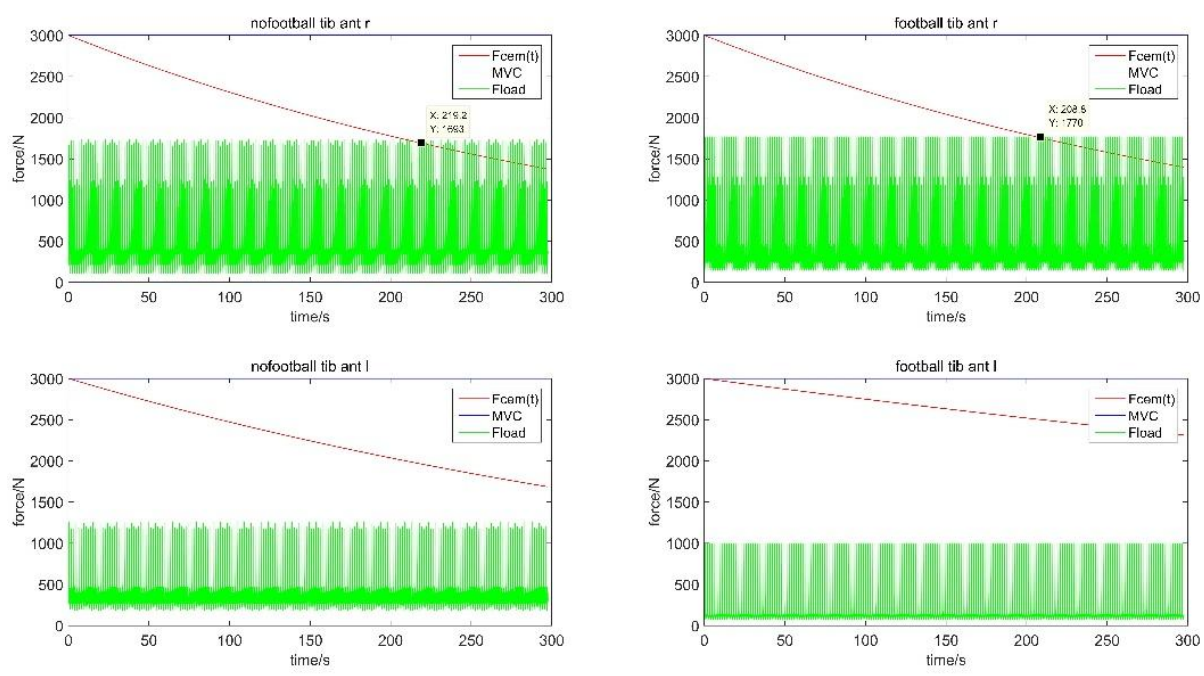

Figure 5. The fatigue process of right and left tib_ant on the calf. 

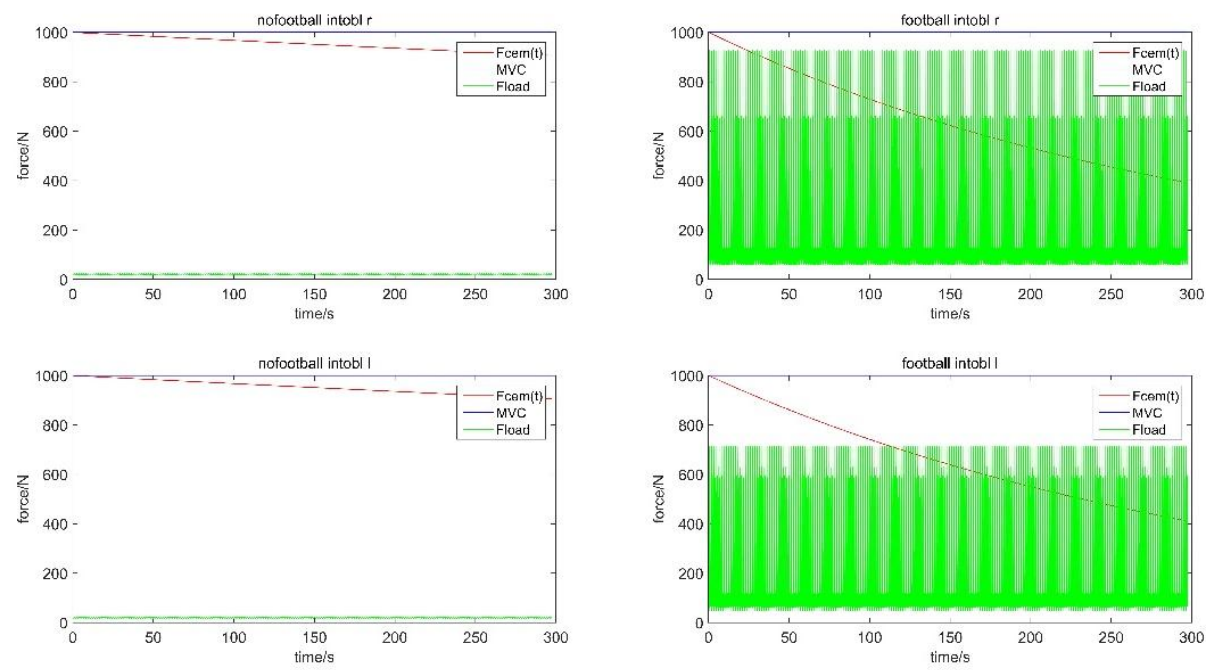

Figure 6. The fatigue process of right and left intobl on the trunk.

The fatigue process in 200 repetitive football-kicking motions of the four typical muscles are shown in Figure 3-6. Red line indicates the muscle force ability while green lines indicates the muscle force requirement at each moment. The intersection of the two indicates the time when current existing muscle force ability declines to the requirement of the motion, so-called the maximal endurance time (MET). After that, the muscle would enter into a risky situation for overuse injuries. It can be found from the figures that the right iliacus in kicking-with-football motion has the shortest MET $(12.39 \mathrm{~s}$, about 8 cycles), followed by the right introbl in kicking-with-football motion (22.85s, about 15 cycles).

\section{Discussion}

\subsection{Contribution of different groups of muscle in the football-kicking motion}

The first surprising result about muscle loads in kicking motion is that the hip muscle group is highly activated (more than $30 \%$ on average). This result may explain the high incidence of hip and groin injury in football players [12-15]. In fact, the hip and groin injury is reported to be the most common non-time-loss injury in football players [13]. Results of this study also show that the loads of hip muscles present little difference between the swing side and supporting side, or between motions with and without football, which indicates that the hip mainly contributes to posing and stabilizing the legs instead of accelerating the football. Besides, the calf muscles, although near the acting point of football reaction force, are not highly activated. The result 
emphasizes the importance of a holistic view in examining workloads in ergonomics [11].

The comparison between simulated muscle loads of the kicking motion with and without football emphasizes the importance of core muscles for football players. The trunk muscle activation is the only that aggravates significantly with the presence of football (about $2 \%$ vs. $24 \%$ ), which indicates that it is the main muscle group contributing to football acceleration. Therefore, stronger trunk muscles may generate better kicking performance. This result expands our previous knowledge about the relationship between strength and football velocity [17-18], from whom a new strategy for football kicking training may come out.

\subsection{Fatigue process for football-kicking training}

The fatigue process analysis of the typical muscles has outlined the fragile muscles, in which the iliacus of the hip proves to be at the highest risk of overuse injury with only height continuous football-kicking exercises sustainable. This muscle is responsible for stabilizing the pelvis in contralateral hip extension and posing the legs [19]. Another muscle at high risk for overuse injury is the internal obliques of the trunk muscle group, with about 15 football-kicking exercises sustainable. Previous research has shown that a larger core muscle reduces the number of missed matches due to injury [20].

In summary, results of this study suggest that the strength training for the hip and the core should be ahead of specific football-kicking training to prevent injuries.

\section{Conclusions}

In this study, we examine the muscle behavior in kicking motion with and without football using OpenSim, then evaluate the fatigue process and MET of repetitive football-kicking training by applying the Dynamic Muscle Fatigue model. It is concluded that muscles of the hip and the knee joints are highly activated in kicking motion, and the football acceleration force mainly depends on the trunk muscles. Muscle at the highest risk for fatigue and overuse injury is the iliacus of the hip joint, which, according to the settings of this study, sustains only height continuous footballkicking cycles. The results may offer a reference for the coaches, athletes, and physiotherapists about the football-kicking training and related injuries.

\section{References}

1. Junge A, Dvorak J. Soccer injuries. Sports medicine, 2004, 34(13): 929-938.

2. Nielsen A B, Yde J. Epidemiology and traumatology of injuries in soccer. The American Journal of Sports Medicine, 1989, 17(6): 803-807.

3. Klünder K B, Rud B, Hansen J. Osteoarthritis of the hip and knee joint in retired football players. Acta orthopaedica Scandinavica, 1980, 51(1-6): 925-927. 
4. Lindberg H, Roos H, Gärdsell P. Prevalence of coxarthrosis in former soccer players: 286 players compared with matched controls. Acta orthopaedica Scandinavica, 1993, 64(2): 165-167.

5. Räty H P, Kujala U M, Videman T, et al. Lifetime musculoskeletal symptoms and injuries among former elite male athletes. International journal of sports medicine, 1997, 18(08): 625-632.

6. Delp S L, Anderson F C, Arnold A S, et al. OpenSim: open-source software to create and analyze dynamic simulations of movement. IEEE transactions on biomedical engineering, 2007, 54(11): 1940-1950.

7. Hamner S R, Seth A, Delp S L. Muscle contributions to propulsion and support during running. Journal of biomechanics, 2010, 43(14): 2709-2716.

8. Chang J, Chablat D, Bennis F, et al. A full-chain OpenSim model and its application on posture analysis of an overhead drilling task[C]//International Conference on HumanComputer Interaction. Springer, Cham, 2019: 33-44.

9. Chang J, Wang W, Chablat D, et al. Evaluating the Effect of Crutch-using on Trunk Muscle Loads[C]//International Conference on Human-Computer Interaction. Springer, Cham, 2020: 455-466.

10. Ma L, Chablat D, Bennis F, et al. A new simple dynamic muscle fatigue model and its validation. International journal of industrial ergonomics, 2009, 39(1): 211-220.

11. Chang J. The risk assessment of work-related musculoskeletal disorders based on OpenSim. PhD Thesis École centrale de Nantes, 2018.

12. Werner J, Hägglund $M$, Waldén $M$, et al. UEFA injury study: a prospective study of hip and groin injuries in professional football over seven consecutive seasons. British journal of sports medicine, 2009, 43(13): 1036-1040.

13. Langhout R, Weir A, Litjes W, et al. Hip and groin injury is the most common non-timeloss injury in female amateur football. Knee Surgery, Sports Traumatology, Arthroscopy, 2019, 27(10): 3133-3141.

14. Werner J, Hägglund M, Waldén M, et al. UEFA injury study: a prospective study of hip and groin injuries in professional football over seven consecutive seasons. British journal of sports medicine, 2009, 43(13): 1036-1040.

15. Feeley B T, Powell J W, Muller M S, et al. Hip injuries and labral tears in the national football league. The American journal of sports medicine, 2008, 36(11): 2187-2195.

16. Nesser T W, Huxel K C, Tincher J L, et al. The relationship between core stability and performance in division I football players. The Journal of Strength \& Conditioning Research, 2008, 22(6): 1750-1754.

17. McGuigan M R, Wright G A, Fleck S J. Strength training for athletes: does it really help sports performance?. International journal of sports physiology and performance, 2012, 7(1): 2-5.

18. Young W B, Rath D A. Enhancing foot velocity in football kicking: the role of strength training. The Journal of Strength \& Conditioning Research, 2011, 25(2): 561-566.

19. Andersson E, Oddsson L, Grundström H, et al. The role of the psoas and iliacus muscles for stability and movement of the lumbar spine, pelvis and hip. Scandinavian journal of medicine \& science in sports, 1995, 5(1): 10-16.

20. Hrysomallis C. Injury incidence, risk factors and prevention in Australian rules football. Sports medicine, 2013, 43(5): 339-354. 